\title{
Société suisse de neurochirurgie
}

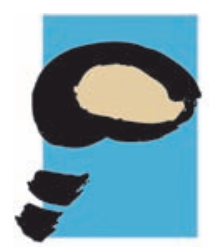

SCHWEIZERISCHE GESELLSCHAFT FÜR NEUROCHIRURGIE SOCIETE SUISSE DE NEUROCHIRURGIE

SOCIETA SVIZZERA DI NEUROCHIRURGIA SWISS SOCIETY OF NEUROSURGERY

www.swissneurosurgery.ch

\section{Raoul Heilbronner}

Président de la SSNC
La Société suisse de neurochirurgie (SSNC) a été fondée en 1954. Présidée à ses débuts par Hugo Krayenbühl, elle a ensuite élu à sa tête Gazi Yasargil, neurochirurgien à la clinique universitaire de neurochirurgie de Zurich, lequel était l'un des plus éminents spécialistes du siècle dernier. La SSNC compte actuellement 93 membres ordinaires dont la moitié environ est établi en cabinet privé.

Chaque année, la SSNC organise deux sessions administratives pour ses membres. Celle du printemps est complétée par une formation postgraduée et continue portant sur des thèmes majeurs de sa discipline; celle d'automne consiste en l'assemblée annuelle tenue avec une autre société apparentée.

Les intérêts des neurochirurgiens en formation sont défendus en son sein par la Swiss Young Neurosurgeons (SYNS), fondée en 2005.

Le comité de la SSNC est actuellement le suivant:

- président: Raoul Heilbronner (SG)

- président désigné: Adrian Merlo (BS)

- secrétaire: Marc Morard (VS)

- secrétaire désigné: Michael Reinert (BE)

- commission de la formation continue: Hans Landolt (AG)

- délégué des neurochirurgiens établis en cabinet privé: Oliver Hausmann (LU)

\section{Affiliations de la SSNC}

La SSNC est membre des associations européenne et mondiale de neurochirurgie (EANS, WFNS). Son affiliation à la FMCH est primordiale sur le plan de la politique professionnelle: compte tenu de sa petite taille, c'est en s'alliant à d'autres sociétés orientées sur la chirurgie qu'elle peut communiquer le mieux ses besoins.

En 2005, elle a participé à la création, à St-Gall, du groupe d'intérêt du personnel opératoire en neurochirurgie de Suisse (IG-NOPPS) et elle y occupe un siège au sein du comité. En contrepartie, elle invite régulièrement les membres IG-NOPPS à sa session d'automne.

Cette année, les membres de la SSNC ont décidé d'adhérer à la Swiss Neuro Federation (SNF), créée en 2008 à Montreux dans le cadre du centième anniversaire de la Société suisse de neurologie. La SNF a pour but de promouvoir la collaboration interdisciplinaire et la politique professionnelle parmi les spécialistes en neuroscience travaillant en milieu hospitalier.

\section{Activités de la SSNC}

La SSNC a pour but de défendre les intérêts de la neurochirurgie en Suisse. Dans ce contexte, la formation postgraduée et continue, le contrôle de la qualité et la garantie de fournir des prestations neurochirurgicales de haute qualité à la population sont des éléments de première importance.

Alors que les travaux du comité étaient accomplis par une à deux personnes il y a encore peu, ils peuvent l'être aujourd'hui uniquement grâce à la collaboration active de plusieurs personnes et à la professionnalisation du secrétariat. Le soutien accordé par la FMCH en faveur de ses activités de politique professionnelle joue également un grand rôle.

Aujourd'hui, la SSNC se voit confrontée à des questions d'une complexité croissante, concernant notamment l'autonomisation accrue de la chirurgie spinale, l'introduction des DRG ou les débats sur la médecine de pointe. 